\title{
Plant Species Richness on a Transmission Right-of-Way in Southeastern Pennsylvania, U.S. Using Integrated Vegetation Management
}

\author{
Richard H. Yahner, Richard T. Yahner, and Bradley D. Ross
}

\begin{abstract}
The Green Lane Research and Demonstration Area, Montgomery County, Pennsylvania, U.S., has been studied each year since 1987, making this 21-year-old project extremely valuable as a source of information on the effects of mechanical and herbicidal maintenance on flora and fauna along an electric transmission right-of-way (ROW). In this article, our objective was to document plant species richness among treatment units and in relation to wire and border zones on the ROW. We noted the presence of plant species from late May through mid-August in 2005 and 2006 and observed 114 vascular plant species in the ten treatment units; $35(31 \%)$ of these species were exotic. Twenty-seven $(24 \%)$ species were widespread on the ROW and seven of these $(26 \%)$ were exotic. The total number of species per unit ranged from $46(40 \%)$ species in handcut units to $57(50 \%)$ in stem-foliage spray units. Of the total number of species, $103(90 \%)$ and $81(71 \%)$ occurred in wire and border zones, respectively. The number of exotic species was higher in wire zones (33 [29\%]) than in border zones (22 [19\%]).

Key Words. Exotic species; herbicides; integrated vegetation management; rights-of-way; southeastern Pennsylvania; species richness; tree control; vegetation.
\end{abstract}

A major goal of the Green Lane 33 Research and Demonstration Project in southeastern Pennsylvania, U.S. since its establishment in 1987 has been to determine the use of Integrated Vegetation Management (IVM) for maintenance of an electric utility transmission right-of-way (ROW) (Bramble and Byrnes 1996; Yahner and Hutnik 2004a). The response of vegetation to IVM is important because plant communities can change within a relatively short time because of natural plant succession.

The IVM technique for vegetation involves two phases: 1) use of a herbicidal spray or mechanical treatment to initially control the density of target (undesirable) trees, i.e., those that have the potential of growing to a height that is not compatible with safe ROW maintenance; and 2) development of a tree-resistant plant cover type to reduce target tree invasion of the ROW (Bramble and Byrnes 1996; Yahner and Hutnik 2004a). The wire-border zone method was used on all treatment units of the Green Lane Research and Demonstration ROW to achieve this goal (Yahner and Hutnik 2004a, 2005; Ballard et al. 2007).

The Green Lane Research and Demonstration Area has been studied each year since 1987, making this 21-year-old project extremely valuable as a source of information on the effects of mechanical and herbicidal maintenance on flora and fauna along an electric transmission ROW (Yahner and Hutnik 2004a, 2004b). A desired objective of IVM on the Green Lane Research and Demonstration Area is to create a diversity of plant species, which in turn provides a diverse wildlife habitat (Yahner and Hutnik 2004a). On a broad scale, habitat diversity on the ROW is achieved by herbicidal and mechanical maintenance of treatment units through the wire-border zone method. However, on a smaller scale, plant species diversity also is achieved by the presence of different combinations of shrub, forb, and grass cover types.
In this article, our objective was to document plant species richness among treatment units and in relation to wire and border zones on the Green Lane Research and Demonstration ROW. Species richness is a simple, yet important, measurement of species diversity and is the total number of different species in a given area (Yahner 2000).

\section{STUDY AREA AND METHODS}

The study area consists of 10 units with two replicates of each of five treatments (mowing, mowing plus herbicide, stem-foliage spray, foliage spray, and handcutting) (Table 1) (see Yahner 2006 for details of treatments). The total area of the ROW is 5.43 ha. Each treatment unit is rectangular in shape, ranges from 0.38 to 2.47 ac (0.15 to $1.00 \mathrm{ha})$, and spans the width of the ROW. The western border zone ( $7 \mathrm{~m}$ wide) of the ROW is immediately adjacent to a ROW of Pennsylvania Power \& Light Company (PP\&L); thus, vegetation was measured only along eastern border zones (11.5 m [37.95 ft] wide). As part of the vegetation assessment of treatment units of plant species from late May through mid-August in both 2005 and 2006, presence of each plant species was noted relative to wire versus border zone in each treatment unit. We used correlation analyses to determine if either the total number of species or the number of exotic species was correlated with size of units (Sokal and Rohlf 1995).

\section{RESULTS AND DISCUSSION}

We observed 114 vascular plant species in 10 treatment units on the Green Lane Research and Demonstration ROW during 2005 to 2006 (Table 1; Appendix). This total number of species at Green Lane was less than the 125 species noted along a transmission ROW in central Pennsylvania (State Game Lands [SGL] 
Table 1. Total number of plant species in wire zones, in border zones, and in either zone of ten treatment units at the Green Lane Research and Demonstration Area from late May through mid-August 2005 to 2006. ${ }^{2}$

\begin{tabular}{|c|c|c|c|c|}
\hline \multirow[b]{2}{*}{ Treatment } & \multirow{2}{*}{$\begin{array}{l}\text { Replicate } \\
\text { unit }\end{array}$} & \multicolumn{3}{|c|}{ Zone } \\
\hline & & Wire & Border & Either \\
\hline \multirow[t]{3}{*}{ Mowing } & M-1 & $52(18,0.36)$ & $35(10,0.24)$ & $56(19,0.60)$ \\
\hline & $M-2^{y}$ & $35(10,0.23)$ & $26(7,0.15)$ & $41(12,0.38)$ \\
\hline & Average & $44(14,0.30)$ & $31(9,0.20)$ & $49(16,0.49)$ \\
\hline \multirow{3}{*}{$\begin{array}{l}\text { Mowing plus } \\
\text { herbicide }\end{array}$} & MH-1 & $33(14,0.19)$ & $36(6,0.13)$ & $52(17,0.32)$ \\
\hline & MH-2 & $34(10,0.60)$ & $28(9,0.40)$ & $48(15,1.00)$ \\
\hline & Average & $34(12,0.40)$ & $32(8,0.27)$ & $50(16,0.66)$ \\
\hline \multirow{3}{*}{$\begin{array}{l}\text { Stem-foliage } \\
\text { spray }\end{array}$} & $\mathrm{SF}-1^{\mathrm{y}}$ & $39(10,0.37)$ & $30(6,0.25)$ & $47(11,0.62)$ \\
\hline & SF-2 & $60(20,0.41)$ & $34(9,0.28)$ & $66(22,0.69)$ \\
\hline & Average & $50(15,0.39)$ & $32(8,0.27)$ & $57(17,0.66)$ \\
\hline \multirow{3}{*}{$\begin{array}{r}\text { Foliage } \\
\text { spray }\end{array}$} & F-1 & $48(15,0.23)$ & $43(10,0.16)$ & $59(16,0.39)$ \\
\hline & $\mathrm{F}-2$ & $34(5,0.35)$ & $28(5,0.24)$ & $41(7,0.59)$ \\
\hline & Average & $41(10,0.29)$ & $36(8,0.20)$ & $50(12,0.49)$ \\
\hline \multirow[t]{3}{*}{ Handcut } & HC-1 & $36(12,0.09)$ & $24(7,0.06)$ & $43(13,0.15)$ \\
\hline & $\mathrm{HC}-2$ & $43(9,0.41)$ & $28(4,0.28)$ & $48(9,0.69)$ \\
\hline & Average & $40(11,0.25)$ & $26(6,0.17)$ & $46(11,0.42)$ \\
\hline $\begin{array}{l}\text { Total area } \\
\text { sampled (ha) }\end{array}$ & & 3.24 & 2.19 & 5.43 \\
\hline
\end{tabular}

${ }^{\mathrm{z}}$ Number of exotic species and total area (ha) are given in parentheses.

${ }^{y}$ Unit adjacent to a secondary road (Upper Ridge Road).

33), but the SGL 33 ROW consisted of 15 units (Yahner and Hutnik 2005).

Total number of species per unit at the Green Lane Research and Demonstration ROW ranged from $46(40 \%)$ in handcut units to 57 (50\%) in stem-foliage units (Table 1; Appendix). Only 27 (24\%) of the total number of species were considered widespread (e.g., those found in $80 \%$ or more of the units), and seven of these (26\%) were exotic. In comparison, $35(31 \%)$ of the total number of species were exotic. This percentage of exotic species is considerably higher than that noted on the SGL 33 ROW $(23 \%)$, which traverses a relatively pristine forested landscape (Yahner and Hutnik 2005), or that found in unfragmented forest stands in Wisconsin (20\%) (Rooney et al. 2004). The Green Lane ROW traverses residential areas that have been humanimpacted for decades; thus, the number of exotic species was expected to be high on the Green Lane ROW, like in a sector of Valley Forge National Historical Park (34\%) in southeastern Pennsylvania (Cypher et al. 1986; see also Lundgren et al. 2004).

Of the total number of species, $103(90 \%)$ and $81(71 \%)$ were in wire and border zones, respectively (Appendix). In wire zones, number of species ranged from $34(30 \%)$ in mowing plus herbicide units to $50(44 \%)$ in stem-foliage units (Table 1). In border zones, number of species varied from $26(23 \%)$ in handcut units to $36(32 \%)$ in foliage-stem units. The number of exotic species ranged from $33(29 \%)$ in wire zones to $22(19 \%)$ in border zones (Appendix). The total number of species or number of exotic species were not correlated with size of units $(r=0.19$ and 0.08 , respectively; $P>0.90$ ). Also, because exotic species were found throughout the ROW, the proximity to a road was not a factor in determining the current distribution of exotic species (Brothers and Spingarn 1992; Watkins et al. 2003; Yahner and Hutnik 2005).

Acknowledgments. Cooperators were Asplundh Expert Tree Company, Dow AgroSciences, and Philadelphia Electric Company.

\section{LITERATURE CITED}

Ballard, B.D., K.T. McLoughlin, and C.A. Nowak. 2007. New diagrams and applications for the wire zone-border zone approach to vegetation management on electric transmission rights-of-way. Arboriculture and Urban Forestry 33:435-439.

Bramble, W.C., and W.R. Byrnes. 1996. Integrated vegetation management of an electric utility right-of-way ecosystem. Down to Earth 51:29-34.

Brothers, T.S., and A. Spingarn. 1992. Forest fragmentation and alien plant invasion of central Indiana old-growth forests. Conservation Biology 6:91-100.

Cypher, E.A., R.H. Yahner, G.L. Storm, and B.L. Cypher. 1986. Flora and fauna survey in a proposed recreational area of Valley Forge National Historical Park. Proceedings of the Pennsylvania Academy of Sciences 60:47-50.

Lundgren, M.R., C.J. Small, and G.D. Dreyer. 2004. Influence of land use and site characteristics on invasive plant abundance in the Quinebaug Highlands of southern New England. Northeastern Naturalist 11:313-322.

Rhoades, A.F., and W.M. Klein Jr. 1993. The Vascular Flora of Pennsylvania: Annotated Checklist and Atlas. American Philosophical Society, Philadelphia, PA. 636 pp.

Rooney, T.P., S.M. Wiegmann, D.A. Rogers, and D.M. Waller. 2004. Biotic impoverishment and homogenization in unfragmented forest understory communities. Conservation Biology 18:787-798.

Sokal, R.R., and F.J. Rohlf. 1995. Biometry: Principles and Practice of Statistics in Biological Research. 3rd Ed. W.H. Freeman and Company, New York, NY. 887 pp.

Watkins, R.Z., J. Chen, J. Pickens, and K.D. Brosofske. 2003. Effects of forest roads on understory plants in a managed forested landscape. Conservation Biology 17:411-419.

Yahner, R.H. 2000. Eastern Deciduous Forest: Ecology and Wildlife Conservation. 2nd Ed. University of Minnesota Press, Minneapolis, MN. 295 pp.

- 2006. Green Lane Research and Demonstration Project: 20 Years of Continuous Study. Annual Report to Cooperators. 36 pp.

Yahner, R.H., and R.J. Hutnik. 2004a. Integrated vegetation management on an electric transmission right-of-way in Pennsylvania, U.S. Journal of Arboriculture 30:295-300.

- 2004b. State Game Lands 33 Research and Demonstration Project-52 Years of Continuous Study. Annual Report to Cooperators. $33 \mathrm{pp}$.

2005. Plant species richness on an electric transmission rightof-way using integrated vegetation management. Journal of Arboriculture 33:124-130.

Richard H. Yahner (corresponding author)

School of Forest Resources

The Pennsylvania State University

University Park, PA 16802, U.S.

rhy@psu.edu

Richard T. Yahner

202 Amblewood Way

State College, PA 16803, U.S.

Bradley D. Ross

734 Partridge Lane

State College, PA 16801, U.S. 


\section{APPENDIX}

Plant species (in phylogenetic order) noted in wire zones (W), in border zones (B), in both zones (WB), or absent (-) in ten treatment units on the Green Lane Research and Demonstration Area from late May through mid-August 2005 to $2006{ }^{\mathrm{z}}$

\begin{tabular}{|c|c|c|c|c|c|c|c|c|c|c|}
\hline Species & M-1 & M-2 & MH-1 & MH-2 & SF-1 & SF-2 & F-1 & $\mathrm{F}-2$ & $\mathrm{HC}-1$ & $\mathrm{HC}-2$ \\
\hline \multicolumn{11}{|l|}{ Hayscented fern } \\
\hline $\begin{array}{l}\text { Dennstaedtia punctilobula } \\
\text { Sensitive fern }\end{array}$ & - & - & B & - & - & WB & - & - & - & WB \\
\hline $\begin{array}{l}\text { Onoclea sensibilus } \\
\text { Pitch pine }\end{array}$ & WB & $\mathrm{W}$ & WB & - & WB & WB & $\mathrm{W}$ & - & B & WB \\
\hline $\begin{array}{l}\text { Pinus rigida } \\
\text { Redcedar }\end{array}$ & - & - & - & - & - & - & - & $\mathrm{W}$ & - & - \\
\hline $\begin{array}{l}\text { Juniperus virginiana } \\
\text { Virginia pine }\end{array}$ & B & - & B & - & WB & WB & WB & WB & B & $\mathrm{W}$ \\
\hline $\begin{array}{l}\text { Pinus virginiana } \\
\text { Sassafras }\end{array}$ & - & - & - & - & B & $\mathrm{W}$ & - & - & - & - \\
\hline $\begin{array}{l}\text { Sassafras albidum } \\
\text { Columine }\end{array}$ & WB & - & B & B & WB & $\mathrm{W}$ & - & B & WB & $\mathrm{W}$ \\
\hline $\begin{array}{l}\text { Aquilegia vulgaris }{ }^{y} \\
\text { Japanese barberry }\end{array}$ & - & - & - & - & - & $\mathrm{W}$ & - & - & - & - \\
\hline $\begin{array}{l}\text { Berberis thunbergii }{ }^{\mathrm{y}} \\
\text { May-apple }\end{array}$ & - & WB & - & - & - & B & $\mathrm{W}$ & WB & - & WB \\
\hline $\begin{array}{l}\text { Podophyllum peltatum } \\
\text { Bloodroot }\end{array}$ & - & - & B & - & - & - & - & - & - & $\mathrm{W}$ \\
\hline $\begin{array}{l}\text { Sanginaria canadensis } \\
\text { Witchhazel }\end{array}$ & - & - & - & - & - & $\mathrm{W}$ & B & - & - & - \\
\hline $\begin{array}{l}\text { Hamamelis virginiana } \\
\text { American elm }\end{array}$ & $\mathrm{W}$ & - & - & - & - & - & - & B & - & W \\
\hline $\begin{array}{l}\text { Ulmus americana } \\
\text { Hickory }\end{array}$ & - & - & - & - & - & - & B & $\mathrm{W}$ & - & - \\
\hline $\begin{array}{l}\text { Carya spp. } \\
\text { Black walnut }\end{array}$ & WB & WB & WB & - & B & - & B & WB & - & - \\
\hline $\begin{array}{l}\text { Juglans nigra } \\
\text { Northern red oak }\end{array}$ & - & - & - & - & B & - & - & - & - & B \\
\hline $\begin{array}{l}\text { Quercus rubra } \\
\text { Black oak }\end{array}$ & $\mathrm{W}$ & WB & B & $\mathrm{W}$ & $\mathrm{W}$ & WB & WB & WB & - & $\mathrm{W}$ \\
\hline $\begin{array}{l}\text { Quercus velutina } \\
\text { Hornbean }\end{array}$ & - & - & - & - & - & - & - & W & - & - \\
\hline $\begin{array}{l}\text { Carpinus caroliniana } \\
\text { Pokeweed }\end{array}$ & - & - & B & - & - & - & WB & - & W & - \\
\hline $\begin{array}{l}\text { Phytolacca americana } \\
\text { Deptford pink }\end{array}$ & - & - & - & - & B & - & - & - & - & - \\
\hline $\begin{array}{l}\text { Dianthus armeria } \\
\text { Smartweed (Pennsylvania) }\end{array}$ & - & - & - & B & - & B & - & - & - & - \\
\hline $\begin{array}{l}\text { Polygonum pensylvanicum } \\
\text { Mile-a-minute }\end{array}$ & - & - & - & $\mathrm{W}$ & - & - & - & - & - & - \\
\hline $\begin{array}{l}\text { Polygonum perfoliatum }{ }^{\mathrm{y}} \\
\text { Climbing false-buckwheat }\end{array}$ & - & - & - & WB & W & W & - & - & - & W \\
\hline $\begin{array}{l}\text { Polygonum scandens }{ }^{\mathrm{y}} \\
\text { Bouncing bet }\end{array}$ & - & - & - & - & B & - & - & - & - & - \\
\hline $\begin{array}{l}\text { Saporaria officinalis } \\
\text { St. John's wort }\end{array}$ & WB & - & WB & W & - & W & $\mathrm{W}$ & - & - & - \\
\hline $\begin{array}{l}\text { Hypericum perforatum } \\
\text { Autumn olive }\end{array}$ & - & - & - & - & - & $\mathrm{W}$ & - & - & - & - \\
\hline $\begin{array}{l}\text { Elaeagnus umbellatay } \\
\text { Prickly cucumber }\end{array}$ & W & WB & W & $\mathrm{W}$ & $\mathrm{W}$ & WB & $\mathrm{W}$ & - & WB & $\mathrm{W}$ \\
\hline $\begin{array}{l}\text { Echinocystis lobata } \\
\text { Violet }\end{array}$ & - & - & - & - & B & - & - & - & - & - \\
\hline $\begin{array}{l}\text { Viola spp. } \\
\text { Garlic-mustard }\end{array}$ & B & - & B & - & B & - & B & - & - & - \\
\hline $\begin{array}{l}\text { Alliaria petiolatay } \\
\text { Horseradish }\end{array}$ & W & - & W & WB & WB & WB & WB & B & WB & - \\
\hline Armoracia rusticana $^{y}$ & - & - & W & - & - & - & - & - & - & - \\
\hline
\end{tabular}




\begin{tabular}{|c|c|c|c|c|c|c|c|c|c|c|}
\hline Species & M-1 & M-2 & MH-1 & MH-2 & SF-1 & SF-2 & F-1 & F-2 & HC-1 & $\mathrm{HC}-2$ \\
\hline Early winter-cress & & & & & & & & & & \\
\hline $\begin{array}{l}\text { Barberea verna }{ }^{\mathrm{y}} \\
\text { Field (cow) cress }\end{array}$ & WB & W & WB & $\mathrm{W}$ & WB & WB & WB & - & $\mathrm{W}$ & W \\
\hline $\begin{array}{l}\text { Lepidium campestre } \\
\text { Poor-man's pepper }\end{array}$ & WB & - & - & - & - & - & - & W & W & - \\
\hline $\begin{array}{l}\text { Lepidium virginicum } \\
\text { Deerberry }\end{array}$ & - & - & W & - & - & - & W & - & - & - \\
\hline $\begin{array}{l}\text { Vaccinium stamineum } \\
\text { Whorled loosestrife }\end{array}$ & - & - & - & - & - & W & - & - & - & W \\
\hline $\begin{array}{l}\text { Lysimachia quadrifolia } \\
\text { Serviceberry }\end{array}$ & - & - & $\mathrm{W}$ & - & W & $\mathrm{W}$ & W & - & - & - \\
\hline $\begin{array}{l}\text { Amelanchier arborea } \\
\text { Chokeberry }\end{array}$ & - & W & - & - & - & - & - & - & - & - \\
\hline $\begin{array}{l}\text { Aronia spp. } \\
\text { Hawthorn }\end{array}$ & - & B & - & - & - & - & - & - & W & W \\
\hline $\begin{array}{l}\text { Crataegus spp. } \\
\text { Wild strawberry }\end{array}$ & - & - & - & - & - & W & B & - & $\mathrm{W}$ & - \\
\hline $\begin{array}{l}\text { Fragaria virginiana } \\
\text { Old-field (common) } \\
\text { cinquefoil }\end{array}$ & W & WB & - & - & - & WB & WB & $\mathrm{W}$ & WB & WB \\
\hline $\begin{array}{l}\text { Potentilla simplex } \\
\text { Wild black cherry }\end{array}$ & $\mathrm{W}$ & WB & WB & B & WB & WB & WB & $\mathrm{W}$ & $\mathrm{W}$ & WB \\
\hline $\begin{array}{l}\text { Prunus serotina } \\
\text { Wild rose }\end{array}$ & $\mathrm{W}$ & WB & B & WB & WB & WB & - & WB & WB & WB \\
\hline $\begin{array}{l}\text { Rosa virginiana } \\
\text { Multiflora rose }\end{array}$ & - & - & - & B & - & - & - & - & - & - \\
\hline $\begin{array}{l}\text { Rosa multiflora } \\
\text { Blackberry }\end{array}$ & WB & WB & B & B & - & WB & WB & WB & WB & WB \\
\hline $\begin{array}{l}\text { Rubus allegheniensis } \\
\text { Swamp dewberry }\end{array}$ & WB & WB & WB & WB & WB & WB & WB & WB & WB & WB \\
\hline $\begin{array}{l}\text { Rubus hispidus } \\
\text { Crown-vetch }\end{array}$ & WB & WB & WB & W & WB & WB & WB & WB & W & WB \\
\hline $\begin{array}{l}\text { Coronilla varia } \\
\text { Maryland tick-clover } \\
\text { (Tick-trefoil) }\end{array}$ & - & - & - & - & - & - & W & - & - & - \\
\hline $\begin{array}{l}\text { Desmodium marilandicum } \\
\text { Tick-trefoil }\end{array}$ & - & - & - & - & W & - & - & - & - & - \\
\hline $\begin{array}{l}\text { Desmodium paniculatum } \\
\text { Virginia (slender) } \\
\text { bush-clover }\end{array}$ & - & - & - & - & - & B & WB & $\mathrm{W}$ & - & - \\
\hline $\begin{array}{l}\text { Lespedeza virginica } \\
\text { Alfalfa (Lucerne alfalfa) }\end{array}$ & - & - & - & - & - & W & - & - & - & - \\
\hline $\begin{array}{l}\text { Medicago sativa } \\
\text { White sweet-clover }\end{array}$ & - & - & - & - & - & W & - & - & - & - \\
\hline $\begin{array}{l}\text { Melilotus alba } \\
\text { Wild bean }\end{array}$ & $\mathrm{W}$ & - & $\mathrm{W}$ & - & - & W & - & - & - & - \\
\hline $\begin{array}{l}\text { Phaseolus polystachios } \\
\text { Red clover }\end{array}$ & - & - & - & - & - & - & B & - & - & - \\
\hline $\begin{array}{l}\text { Trifolium pretense } \\
\text { White clover }\end{array}$ & - & - & - & - & - & - & W & - & - & - \\
\hline $\begin{array}{l}\text { Trifolium repens }{ }^{y} \\
\text { Evening-primrose }\end{array}$ & $\mathrm{W}$ & - & $\mathrm{W}$ & - & - & - & W & - & - & - \\
\hline $\begin{array}{l}\text { Oenothera biennis } \\
\text { Flowering dogwood }\end{array}$ & WB & - & $\mathrm{W}$ & W & - & WB & W & - & - & - \\
\hline $\begin{array}{l}\text { Cornus florida } \\
\text { Round-leaved (gray) } \\
\text { dogwood }\end{array}$ & - & - & - & - & - & B & B & - & B & WB \\
\hline $\begin{array}{l}\text { Cornus rugusa } \\
\text { Oriental (Asiatic) } \\
\text { bittersweet }\end{array}$ & B & WB & B & B & - & $\mathrm{W}$ & $\mathrm{W}$ & WB & $\mathrm{W}$ & WB \\
\hline Celastrus orbiculatus ${ }^{y}$ & - & B & B & - & $\mathrm{W}$ & - & WB & - & B & - \\
\hline
\end{tabular}




\begin{tabular}{|c|c|c|c|c|c|c|c|c|c|c|}
\hline Species & M-1 & M-2 & MH-1 & MH-2 & SF-1 & SF-2 & F-1 & F-2 & HC-1 & HC-2 \\
\hline \multicolumn{11}{|l|}{ Virginia-creeper } \\
\hline $\begin{array}{l}\text { Parthenocissus quinquefolia } \\
\text { Grape }\end{array}$ & W & B & B & B & B & W & WB & WB & WB & WB \\
\hline $\begin{array}{l}\text { Vitis spp. } \\
\text { Red maple }\end{array}$ & WB & WB & - & W & WB & WB & WB & WB & WB & WB \\
\hline $\begin{array}{l}\text { Acer rubrum } \\
\text { Sugar maple }\end{array}$ & - & - & B & W & W & W & W & W & - & WB \\
\hline $\begin{array}{l}\text { Acer saccharum } \\
\text { Staghorn Sumac }\end{array}$ & - & - & - & - & WB & - & WB & B & - & B \\
\hline $\begin{array}{l}\text { Rhus typhina } \\
\text { Poison-ivy }\end{array}$ & - & - & - & - & - & - & B & - & - & - \\
\hline $\begin{array}{l}\text { Toxicodendron radicans } \\
\text { Wood-shamrock } \\
\text { (Wood sorrel) }\end{array}$ & WB & WB & B & WB & WB & W & WB & WB & WB & WB \\
\hline $\begin{array}{l}\text { Oxalis dillenii } \\
\text { Wild geranium }\end{array}$ & W & - & WB & WB & W & - & WB & - & W & - \\
\hline $\begin{array}{l}\text { Geranium carolinianum } \\
\text { Jewelweed }\end{array}$ & WB & - & - & - & - & WB & - & - & - & - \\
\hline $\begin{array}{l}\text { Impatiens spp. } \\
\text { Spreading dogbane }\end{array}$ & WB & - & WB & WB & - & - & - & - & W & W \\
\hline $\begin{array}{l}\text { Apocynum } \\
\text { androsaemifolium } \\
\text { Purple milkweed }\end{array}$ & - & - & - & W & - & W & - & - & - & W \\
\hline $\begin{array}{l}\text { Asclepias purpurascens } \\
\text { Common milkweed }\end{array}$ & - & - & - & W & W & - & - & - & - & - \\
\hline $\begin{array}{l}\text { Asclepias syriaca } \\
\text { Field bindweed }\end{array}$ & WB & W & WB & W & W & WB & W & W & WB & WB \\
\hline $\begin{array}{l}\text { Convolvulus arvensis } \\
\text { Phlox }\end{array}$ & WB & - & WB & W & W & WB & - & - & - & - \\
\hline $\begin{array}{l}\text { Phlox spp. } \\
\text { Fleabane }\end{array}$ & - & - & - & - & - & - & - & W & - & - \\
\hline $\begin{array}{l}\text { Erigeron philadelphicus }{ }^{\mathrm{y}} \\
\text { Heal-all }\end{array}$ & WB & - & - & - & - & - & - & - & W & - \\
\hline $\begin{array}{l}\text { Prunella vulgaris } \\
\text { White ash }\end{array}$ & - & - & - & - & - & W & - & - & - & - \\
\hline $\begin{array}{l}\text { Fraxinus americana } \\
\text { Beard-tongue }\end{array}$ & WB & WB & WB & WB & WB & WB & WB & WB & WB & WB \\
\hline $\begin{array}{l}\text { Penstemon hirsutus } \\
\text { Mullein }\end{array}$ & - & - & - & W & - & - & - & - & - & - \\
\hline $\begin{array}{l}\text { Verbascum thapsus } \\
\text { Bedstraw }\end{array}$ & W & W & W & WB & WB & W & WB & - & WB & W \\
\hline $\begin{array}{l}\text { Galium spp. } \\
\text { Japanese honeysuckle }\end{array}$ & W & W & B & - & WB & WB & WB & WB & W & W \\
\hline $\begin{array}{l}\text { Lonicera japonica } \\
\text { Tatarian honeysuckle }\end{array}$ & WB & B & B & B & WB & WB & B & WB & WB & WB \\
\hline $\begin{array}{l}\text { Lonicera tatarica } \\
\text { Southern arrowwood } \\
\text { (Viburnum) }\end{array}$ & WB & WB & - & B & - & W & - & B & WB & - \\
\hline $\begin{array}{l}\text { Viburnum dentatum } \\
\text { Black-haw }\end{array}$ & - & WB & B & B & WB & WB & - & WB & B & - \\
\hline $\begin{array}{l}\text { Viburnum prunifolium } \\
\text { Common yarrow }\end{array}$ & WB & WB & WB & $\mathrm{B}$ & WB & W & - & WB & B & W \\
\hline $\begin{array}{l}\text { Achillea millefolium } \\
\text { Common ragweed }\end{array}$ & W & WB & W & WB & W & WB & WB & WB & W & W \\
\hline $\begin{array}{l}\text { Ambrosia artemisiifolia } \\
\text { Dog-fennel (Mayweed) }\end{array}$ & - & - & - & - & - & - & - & - & - & B \\
\hline $\begin{array}{l}\text { Anthenis colula } \\
\text { Tickseed-sunflower }\end{array}$ & WB & - & W & - & WB & - & WB & - & W & - \\
\hline $\begin{array}{l}\text { Bidens aristosa } \\
\text { Sheep sorrel }\end{array}$ & - & - & - & WB & - & $\mathrm{W}$ & - & - & - & - \\
\hline Rumex acetosella ${ }^{y}$ & - & - & - & W & - & $\mathrm{W}$ & - & - & - & - \\
\hline
\end{tabular}




\begin{tabular}{|c|c|c|c|c|c|c|c|c|c|c|}
\hline Species & M-1 & M-2 & MH-1 & MH-2 & SF-1 & SF-2 & F-1 & F-2 & $\mathrm{HC}-1$ & HC-2 \\
\hline $\begin{array}{l}\text { Curly (curled) dock } \\
\text { Rumex crispus }{ }^{y} \\
\text { Thistle (several species) }\end{array}$ & W & - & W & - & - & - & - & - & W & - \\
\hline $\begin{array}{l}\text { Circium spp. } \\
\text { Bird's-eye speedwell }\end{array}$ & WB & - & W & B & W & W & W & WB & W & W \\
\hline $\begin{array}{l}\text { Veronica persica } a^{\mathrm{y}} \\
\text { American elder (elderberry) }\end{array}$ & W & - & W & - & - & - & WB & - & - & - \\
\hline $\begin{array}{l}\text { Sambucus canadensis } \\
\text { Hysop-leaved thoroughwort }\end{array}$ & W & WB & - & - & - & - & WB & - & - & WB \\
\hline $\begin{array}{l}\text { Eupatorium hyssopifolium } \\
\text { Boneset }\end{array}$ & - & - & - & W & - & W & - & - & - & $\mathrm{W}$ \\
\hline $\begin{array}{l}\text { Eupatorium perfoliatum } \\
\text { Joe-pie-weed }\end{array}$ & WB & W & W & - & - & B & WB & - & - & - \\
\hline $\begin{array}{l}\text { Eupatorium purpureum } \\
\text { Orange hawkweed }\end{array}$ & - & - & - & - & - & - & - & - & - & W \\
\hline $\begin{array}{l}\text { Hieracium aurantiacum } \\
\text { King-devil (Smoothish } \\
\text { hawkweed) }\end{array}$ & - & W & - & - & - & - & - & - & - & - \\
\hline $\begin{array}{l}\text { Hieracium piloselloides }{ }^{y} \\
\text { Hawkweed }\end{array}$ & - & W & - & - & - & W & W & - & - & - \\
\hline $\begin{array}{l}\text { Hieracium scabrum } \\
\text { Wild lettuce }\end{array}$ & W & W & - & - & - & - & WB & W & - & - \\
\hline $\begin{array}{l}\text { Lactuca canadensis } \\
\text { Black-eyed-susan }\end{array}$ & W & - & - & - & - & - & - & - & W & - \\
\hline $\begin{array}{l}\text { Rudbeckia hirta } \\
\text { Golden ragwort }\end{array}$ & - & - & - & - & - & B & - & - & - & - \\
\hline $\begin{array}{l}\text { Senecio aureus } \\
\text { Goldenrod (several species) }\end{array}$ & B & - & - & - & - & W & - & - & - & \\
\hline $\begin{array}{l}\text { Solidago spp. } \\
\text { Milk-thistle (Common } \\
\text { sow-thistle) }\end{array}$ & WB & WB & WB & WB & WB & WB & WB & WB & WB & WB \\
\hline $\begin{array}{l}\text { Sonchus oleraceus } \\
\text { Yellow goat's-beard }\end{array}$ & - & - & - & W & - & W & - & - & - & - \\
\hline $\begin{array}{l}\text { Tragopogon pratensis }^{y} \\
\text { Jack-in-the-pulpit }\end{array}$ & W & - & W & - & - & - & W & - & - & - \\
\hline $\begin{array}{l}\text { Arisaema triphyllum } \\
\text { Sedge }\end{array}$ & WB & - & B & B & WB & WB & B & B & - & WB \\
\hline $\begin{array}{l}\text { Carex spp. } \\
\text { Wild oats }\end{array}$ & WB & W & WB & W & - & - & WB & - & WB & B \\
\hline $\begin{array}{l}\text { Avena fatua }^{y} \\
\text { Deer-tongued grass }\end{array}$ & WB & - & - & - & - & - & WB & - & - & - \\
\hline $\begin{array}{l}\text { Panicum clandestinum } \\
\text { Fall panic grass }\end{array}$ & WB & - & B & W & - & - & WB & W & W & - \\
\hline $\begin{array}{l}\text { Panicum spp. } \\
\text { Common reed (Phragmites) }\end{array}$ & WB & B & WB & WB & WB & WB & WB & B & B & WB \\
\hline $\begin{array}{l}\text { Phagmites australis } \\
\text { Field garlic }\end{array}$ & W & W & WB & W & W & W & - & - & - & W \\
\hline $\begin{array}{l}\text { Allium oleraceum } \\
\text { False Solomon's seal }\end{array}$ & - & W & - & - & - & W & - & - & - & WB \\
\hline $\begin{array}{l}\text { Smilacina trifolia } \\
\text { White twisted-stalk }\end{array}$ & - & W & - & B & WB & WB & WB & WB & W & - \\
\hline $\begin{array}{l}\text { Streptopus amplexifolius } \\
\text { Blue-eyed grass }\end{array}$ & - & - & - & - & - & W & - & - & - & - \\
\hline $\begin{array}{l}\text { Sisyrinchium angustifolium } \\
\text { Carrion-flower }\end{array}$ & - & W & - & WB & - & - & - & W & - & - \\
\hline $\begin{array}{l}\text { Smilax herbaea } \\
\text { Greenbriar }\end{array}$ & WB & - & - & - & W & - & - & - & - & - \\
\hline Smilax rotundifolia & - & B & - & - & $\mathrm{W}$ & WB & B & - & - & B \\
\hline
\end{tabular}

${ }^{\mathrm{z}}$ The replication of each treatment is given after each unit, where treatments are mowing $(\mathrm{M})$, mowing plus herbicide $(\mathrm{MH})$, stem-foliage spray (SF), foliage spray $(\mathrm{F})$, and handcut (HC). Common and scientific names are taken from Rhoades and Klein (1993).

${ }^{\mathrm{y}}$ Considered an exotic species (Rhoades and Klein 1993). 
Résumé. L'Aire de recherche et de démonstration de corridor vert $\mathrm{du}$ comté de Montgomery en Pennsylvanie a été étudiée annuellement depuis 1987, ce qui fait de ce projet vieux de 21 ans une source précieuse d'information sur les effets des traitements mécaniques et chimiques d'entretien par rapport à la flore et la faune le long des emprises de lignes électriques de transport. Dans cet article, notre objectif était de documenter la richesse en espèces de plantes parmi les unités traités en relation avec la zone à l'intérieur de l'emprise et la zone limitrophe à l'emprise. Nous avons noté la présence d'espèces de plantes de la fin mai jusqu'à la mi-août en 2005 et 2006 et y avons observé la présence de 114 espèces de plantes vasculaires au sein des 10 unités de traitement; $35(31 \%)$ de ces espèces étaient non indigènes. Vingt-sept $(24 \%)$ espèces se retrouvaient au sein de toutes les unités dont 7 (26\%) d'entre elles étaient non indigènes. Le nombre total d'espèces par unité était en moyenne de $46(40 \%)$ au sein des unités traitées mécaniquement et de $57(50 \%)$ au sein des unités traitées chimiquement. Du nombre total d'espèces, $103(90 \%)$ étaient présentes à l'intérieur de l'emprise tandis que $81(71 \%)$ étaient présentes dans la zone limitrophe à l'emprise. Le nombre d'espèces exotiques était plus élevé à l'intérieur de l'emprise (33 espèces ou 29\%) qu'en bordure de l'emprise (22 espèces ou $19 \%)$.

Zusammenfassung. Die Green Lane Forschungs- und Demonstrationsfläche in Montgomery County, Pennsylvanien wird seit 1987 jährlich untersucht. Dadurch ist dieses 21jährige Experiment eine extrem wertvolle Informationsquelle für den Einfluss von mechanischer und chemischer Kontrolle von Flora und Fauna entlang einer Überlandleitung. In dieser Studie war unser Ziel, die Pflanzenartenvielfalt in den Behandlungseinheiten und in Relation zu Zentrum und Randzonen zu dokumentieren. Wir erfassten die gegenwärtigen Pflanzenarten von
Ende Mai bis Mitte August 2005 und 2006 und beobachteten dabei 114 vaskuläre Pflanzenarten in 10 Behandlungseinheiten. 35 (31\%) dieser Arten waren exotisch. 27 (24\%) waren bei Überlandleitungen weit verbreitet und 7 von diesen (26\%) waren exotisch. Die totale Anzahl von Arten pro Einheit betrug durchschnittlich $46(40 \%)$ in handgeschnittenen Einheiten und 57 (50\%) in Einheiten mit Spray-Herbizideinsatz. Von der totalen Artenanzahl tauchten 103 (90\%) im Zentrum unter den Drähten auf und 81 (71\%) in den Randbereichen. Die Anzahl der exotischen Arten war im Zentrum (33, 29\%) höher als in den Randbereichen $(22,19 \%)$.

Resumen. El área de demostración de Green Lane Research, Montgomery County, Pennsylvania, U.S., ha sido estudiada anualmente desde 1987, logrando con esto 21 años de proyectos extremadamente valiosos como fuente de información sobre los efectos de las prácticas de mantenimiento de la flora y fauna, mecánicos y con herbicidas, a lo largo de la líneas de transmisión del derecho de vía (ROW, por sus siglas en inglés). En este reporte, nuestro objetivo fue documentar la riqueza de especies de plantas entre unidades de tratamiento y en relación a las zonas de frontera alambradas en el ROW. Se notó la presencia de especies de plantas desde Mayo y a lo largo de Agosto en 2005 y 2006, y se observaron 114 especies de plantas vasculares en las 10 unidades de tratamiento; $35(31 \%)$ de estas especies fueron exóticas. Veintisiete (24\%) estaban ampliamente distribuidas y siete de estas especies $(26 \%)$ fueron exóticas. El número total de especies por unidad promedió de 46 (40\%), en unidades cortadas manualmente, a $57(50 \%)$ en unidades con aerosol. Del número total de especies, $103(90 \%)$ y 81 (71\%) se presentaron en zonas alambradas y de frontera, respectivamente. El número de especies exóticas fue más alto en zonas alambradas (33, 29\%) que en zonas de frontera $(22,19 \%)$. 